\title{
Alkali Cation Attachment to Derivatized Fullerenes Studied by Matrix-Assisted Laser Desorption/Ionization
}

\author{
Dorina Fati, ${ }^{*}$ Victoria Leeman, Yury V. Vasil'ev, and Thomas Drewello \\ Department of Chemistry, University of Warwick, Coventry CV4 7AL, UK
}

\author{
Bernard Leyh $^{+}$ \\ Department of Chemistry, University of Liege, Liege, Belgium
}

\author{
Hartmut Hungerbühler \\ Fachbereich II Pharma- und Chemietechnik, Technische Fachhochschule Berlin, Berlin, Germany
}

\begin{abstract}
The complexation of alkali metal ions with amphiphilic fullerene derivatives has been investigated by matrix-assisted laser desorption/ionization (MALDI) time-of-flight (TOF) mass spectrometry. The formation of analyte ions occurs via two competing mechanisms including electron transfer from matrix-derived ions and metal ion attachment. The interplay of these processes has been examined by laser fluence dependent sample activation and by variation of the target composition. The attachment of metal ions has been established as the gentler and thus more efficient route towards the formation of intact analyte ions. Investigations into the metal ion complexation have been conducted to reveal the reactivity order of the alkali metals in these reactions and to elucidate the influence of structural differences of the analytes, as well as to unravel effects caused by the anionic counter ion of the metal. The experimental data have been derived by two complementary approaches. Competing reactants were either studied simultaneously, so that the product distribution would provide direct insight into the reactivity pattern, and/or product distributions were obtained in a large variety of separate experiments and normalized for reliable comparison. It has been found that the extent to which complexation is observed follows the charge density order of the alkali metal ions. The structural features of the fullerene-attached ligands were of profound influence on the attachment of the metal ion, inducing enhanced selectivity for the complexation with less reactive metals. The metal ion attachment is reduced with the use of smaller anionic counter ions. Rationalization of these findings is provided within the framework of the mechanisms of ion formation in MALDI. (J Am Soc Mass Spectrom 2002, 13, 1448-1458) () 2002 American Society for Mass Spectrometry
\end{abstract}

$\mathrm{T}$ The chemistry of fullerene-based compounds has experienced tremendous developments in recent years $[1,2]$. The spherical fullerene core represents a unique geometrical feature which enables the creation of three-dimensional molecular architecture with tailor-made material properties. Research into potential applications in material sciences is most prominently conducted in areas covering the design of new electronic and optical devices, chemical sensors, and polymers [3]. Water-soluble fullerene derivatives have also paved the way for investigations into biological applications. The effective inhibition of HIV-1 protease,

Published online November 7, 2002

Address reprint requests to Dr. T. Drewello, University of Warwick, Coventry CV4 7AL, UK. E-mail: T.Drewello@warwick.ac.uk

*Also at the Department of Chemistry, University of Liege, Liege, Belgium. tResearch associate of the F.N.R.S. (Belgium) anticancer activity in photodynamic therapy, and the development of tracer compounds for radiotherapy and medical imaging are some examples of fullerene modification for biological uses [4,5]. The compounds studied in this investigation are depicted in Figure 1a and $\mathrm{b}$. In these materials the hydrophobic $\mathrm{C}_{60}$ moiety is methylene-bridged to a long-chained, hydrophilic ligand, which results in molecules with amphiphilic properties. Amphiphilic fullerenes have gained attention as potential biosensors by exploiting their ability to form stable, ordered monomolecular layers and Langmuir-Blodgett films [6, 7].

Most chemical modifications of the fullerene cage lead to multiple ligand additions and to the formation of isomers. Progress in fullerene chemistry has been therefore inevitably connected with the availability of methods for a reliable analysis of trace amounts. In this context, mass spectrometry has been the most success- 
a)

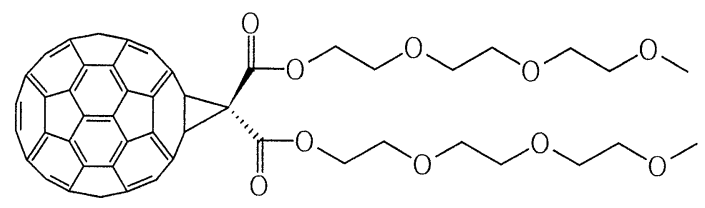

b)

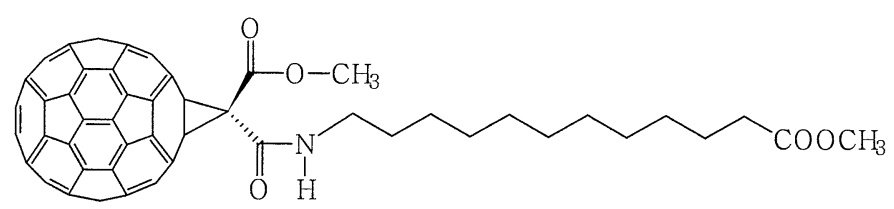

c)

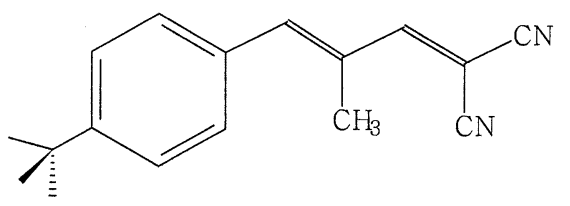

Figure 1. The structures of the amphiphilic fullerene derivatives and the matrix compound (a) Analyte $1(\mathrm{M}=1114 \mathrm{~g} / \mathrm{mol})$, (b) Analyte $2(\mathrm{M}=1047 \mathrm{~g} / \mathrm{mol})$, (c) Matrix, DCTB $(\mathrm{M}=250 \mathrm{~g} / \mathrm{mol})$.

fully applied analytical tool. However, most derivatized fullerenes are thermally labile solids of only low vapor pressure. Therefore the use of more conventional ionization methods is often prevented, as heating is applied to the transfer of the analyte into the gas phase. As a consequence, "soft" ionization methods that allow a gentler gas phase transfer, such as electrospray ionization (ESI) and MALDI, have been more appropriate for the analysis of these materials. However, both ESI and MALDI afford a certain adaptation for the analysis of derivatized fullerenes. The use of ESI has been particularly successful when the experimental parameters were adjusted for using the ESI source as an intrinsic electrochemical cell [8]. Under these conditions, the formation of ions occurs through electrochemical oxidation or reduction $[9,10]$. The use of nanoflow-ESI has reduced the amount of sample required for the analysis [11].

The application of MALDI has been greatly improved through the evaluation of matrix materials suited for the analysis of the respective fullerene-based compounds [11-15]. For most organic fullerene derivatives the ion formation in MALDI seems to involve predominately electron transfer, leading to positive and/or negative molecular ions rather than to protonated and/or deprotonated species.

The present investigation examines in detail ion formation through metal ion attachment to amphiphilic fullerenes. Recent MALDI experiments have revealed that for these compounds metal ion attachment may efficiently compete with electron transfer, even without metal ion doping of the target [16]. To achieve more insight into the attachment process, particular metal ions are deliberately added to the target in the form of a salt layer. Enhancing our understanding of metal ion attachment in MALDI is, in principle, of great analytical value. The MALDI analysis of polymers and dendrimers is almost entirely based on ion formation via the attachment of metal ions to the analyte and the experimental parameters influencing the determination of molecular weight distributions are of current concern [17]. Due to their importance in related MALDI applications, the present approach focuses on the use of the alkali metal ions. The metal ion attachment is examined here by a variety of different MALDI experiments. The metal ions were provided for each fullerene analyte through salt layers of the individual alkali metals. Insight into the ion formation dynamics has been obtained in MALDI experiments at different fluences of the incoming laser light. MALDI of the individual analytes has been conducted applying salt layers with different metal ion combinations. Furthermore, the competition between the two analytes for the attachment of a particular metal ion has been studied using equimolar analyte mixtures. Finally, possible counter 
ion effects have been investigated by variation of the anionic component of the salt.

\section{Experimental}

\section{Instrumentation}

The experiments have been performed using a time-offlight (TOF) mass spectrometer composed of ion source, linear flight tube, and quadratic-field reflectron (Kompact MALDI IV, Kratos Inc., Manchester, UK). Earlier investigations detailing different applications of this instrumental set-up, for the study of fullerenerelated materials, can be found elsewhere [18-21]. The ion source operates a nitrogen laser at an ultraviolet wavelength of $337 \mathrm{~nm}$ with a pulse width of $3 \mathrm{~ns}$ and frequency $1.5 \mathrm{~Hz}$. All mass spectra were recorded in positive-ion-reflectron mode, applying a continuous acceleration voltage of $20 \mathrm{kV}$. For each individual mass spectrum two hundred single laser shot events were accumulated at constant laser fluence. The sample holder was moved prior to each individual laser shot to ensure activation of a pristine target area. The collected data were averaged and viewed in electronically processed form. Calibration of the mass spectra was performed using $C_{60}$ and $C_{70}$ fullerenes. The laser fluence as an experimental parameter will be different for different instrumental setups and is therefore given in arbitrary units (following the Kompact MALDI IV reading) throughout the text.

The error bars plotted in Figures 5, 7, and 8 represent the standard deviation, $\sigma$ of the mean values.

\section{Sample Preparation}

The amphiphilic analytes were synthesized as described in the literature [6] and dissolved in toluene at a ratio of $1 \mathrm{mg}$ analyte per $1 \mathrm{ml}$ of solvent. DCTB (trans-2-[3-\{4-tert-Butylphenyl\}-2-methyl-2-propenylidene]malononitrile, whose structure is shown in Figure 1c) was supplied by Fluka (Buchs, Switzerland) and used as the matrix. DCTB was recently introduced as a matrix material for MALDI with derivatized fullerenes [15] and comprehensive screening experiments including some fourteen common matrices revealed DCTB as the best performing matrix for this purpose [16]. DCTB was dissolved in toluene at a concentration of $1 \mathrm{mg}$ per $\mathrm{ml}$. Both analyte and matrix solution were freshly prepared and combined to a molar matrix-to-analyte ratio of 1000:1 [16]. To produce the salt layers, the alkali halides (Sigma Aldrich, Gillingham-Dorset, UK, 99.9\% purity) were dissolved in methanol as 0.001 molar solutions (for solubility see [22]).

\section{Target Preparation}

The homogeneity over a large surface area of the sample is a major concern regarding reproducibility in MALDI [23]. To prevent unwanted effects caused by inconsistencies of the target preparation, all MALDI targets were routinely prepared in the following way. The sample holders (stainless steel slides) were thoroughly cleaned using dichlorobenzene followed by acetonitrile, acetone, and finally methanol. A layering method, as applied to the MALDI cationization of polymers [17-21, 23, 24], has been adopted. Bottom layers of homogenous appearance were obtained by depositing the salt solutions from a $10 \mu \mathrm{L}$ syringe onto the initially heated sample holder, ensuring quick evaporation of the solvent. The clear white salt layer was allowed to dry completely before the matrix/analyte solution was deposited at room temperature, preventing decomposition of the thermally labile analyte. After fully coating the salt layer, the sample holder was dried in a cold air stream, leaving a dense matrix/analyte layer on top of the salt layer underneath.

\section{Results and Discussion}

\section{Cation Attachment to Fullerene Derivatives}

The laser activation of targets composed of an analyte/ matrix layer on top of an alkali salt layer leads to efficient attachment of the respective alkali metal ion to both fullerene analytes. Typical spectra obtained for Analyte 1 are shown in Figure 2a, b, c, d, and e for the whole series of alkali chloride cations. The appearance of the MALDI spectra of Analyte 2 was very similar.

Alkali metal ion attachment to crown ether-ligated fullerenes has been achieved in earlier ESI experiments by spraying of presumably preformed adduct ions [25]. The present experiments, however, represent the first successful attempt using MALDI to analyze derivatized fullerenes through gas phase metal ion attachment. It can be assumed that attachment occurs predominately through interaction of the fullerene ligand with the metal ion.

In principle, one also has to consider metal attachment to the fullerene core. Complexes of bare metal ions with pure fullerenes are thermodynamically stable species and were in fact experimentally observed [26-28]. However, the stabilization of metal ion/fullerene complexes has been achieved through the release of excess energy during complex formation. However, similar effects can be discounted for the present experiments on the basis that no metal ion attachment could be achieved in MALDI experiments for which the analyte was pure $\mathrm{C}_{60}$. Furthermore, it will be shown later that the structure of the fullerene ligand strongly affects the metal ion attachment, also indicating that the interaction between ligand and metal ion is crucial for the complex formation.

In addition to the desired metal ion/analyte signal, the MALDI spectra (Figure 2a, b, c, d, and e) contain several other features indicative of processes which accompany the attachment. The origin of the signals of the bare metal ions will be addressed in later discussion. The attachment of metal ions that were initially 


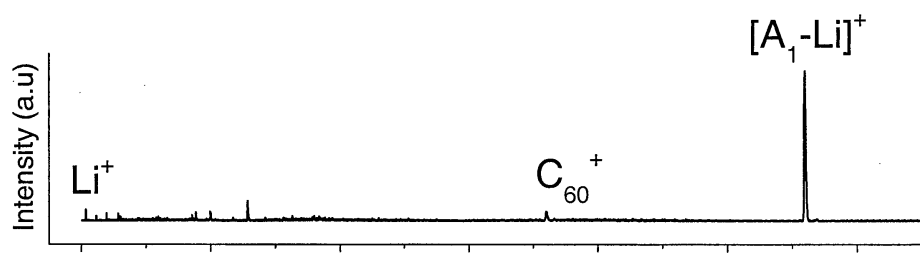

a)

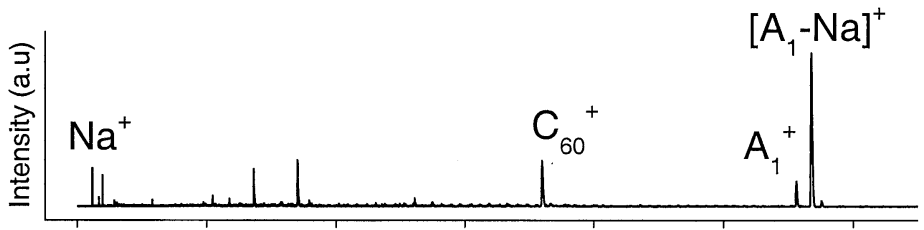

b)

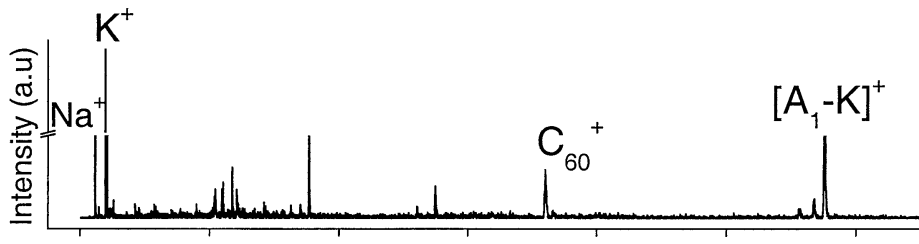

C)

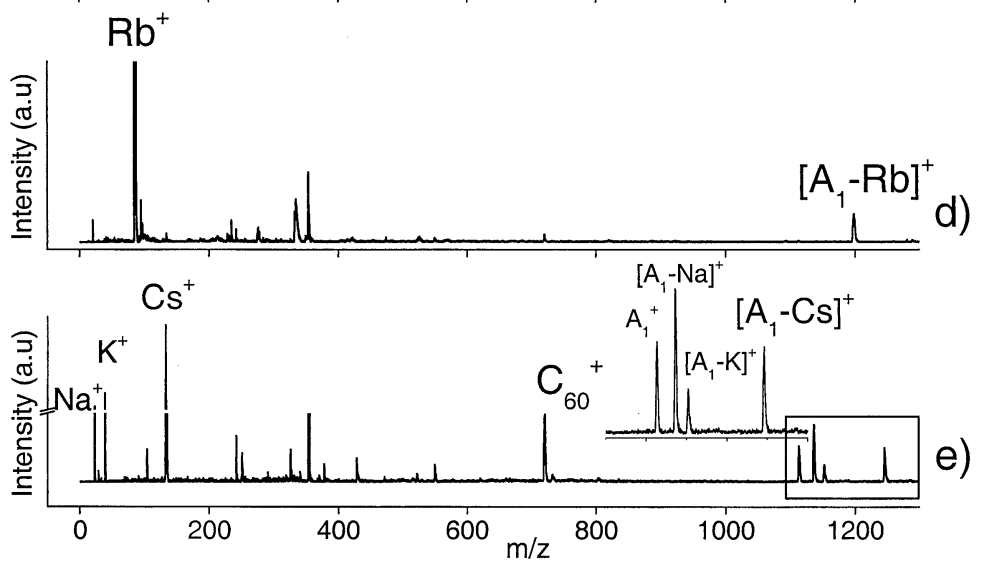

Figure 2. MALDI spectra of the Analyte 1 obtained with different alkali chloride salt layers: (a) LiCl, (b) $\mathrm{NaCl}$, (c) $\mathrm{KCl}$, (d) $\mathrm{RbCl}$, (e) $\mathrm{CsCl}$.

provided through the salt layer has been observed in all experiments. However, in some of the spectra there is clear evidence for the attachment of metals that were not deliberately provided. The intended formation of the sodiated analyte is accompanied by potassium attachment (Figure $2 b$ ) and, vice versa, the anticipated formation of the potassium complex (Figure 2c) shows competition of sodium attachment. Most dramatically seen is this effect on using a cesium salt layer (Figure $2 \mathrm{e})$, revealing the abundant coformation of both sodiated and potassiated analyte complexes. The origin of these metals is either through impurities of the salts, solutions, or glassware. The efficient attachment of metal ions initially available in such minor amounts indicates substantial differences for the efficiency of the attachment of the different metals. Therefore, when considering in later experiments the competition of the different alkali ions, the metal ions were loaded onto the target holder in excess and in equal quantities.

A further intriguing observation is the appearance of the molecular cation radical of the analyte. Unlike the metal ion complexed analyte, this species represents the odd electron molecular ion of the analyte. This ionized analyte is not likely to result as a major fragment from dissociation of the metal ion complexed analyte. Although the ionization energy of the analyte is not known, it can be assumed that it is not substantially different from the ionization energy of $\mathrm{C}_{60}$ itself $(7.6 \mathrm{eV})$ [29] which is substantially higher than the ionization energies of the alkali metals [30] $(\mathrm{IE}[\mathrm{Li}]=5.38 \mathrm{eV}$; $\mathrm{IE}[\mathrm{Na}]=5.12 \mathrm{eV} ; \mathrm{IE}[\mathrm{K}]=4.34 \mathrm{eV} ; \mathrm{IE}[\mathrm{Rb}]=4.16 \mathrm{eV}$; $\mathrm{IE}[\mathrm{Cs}]=3.90 \mathrm{eV}$ ). As a result, charge transfer from the metal ion to the analyte is unlikely to occur. The most likely origin of the molecular cation radical of the analyte is thus through electron transfer involving matrix-derived ions. This in turn provides a unique opportunity to investigate metal ion complexation and electron transfer as the two competing mechanisms for the formation of ions from the same analyte.

\section{Laser Fluence Dependency of Ion Formation}

The threshold behavior of the various ions detailed above has been investigated as a function of the laser 


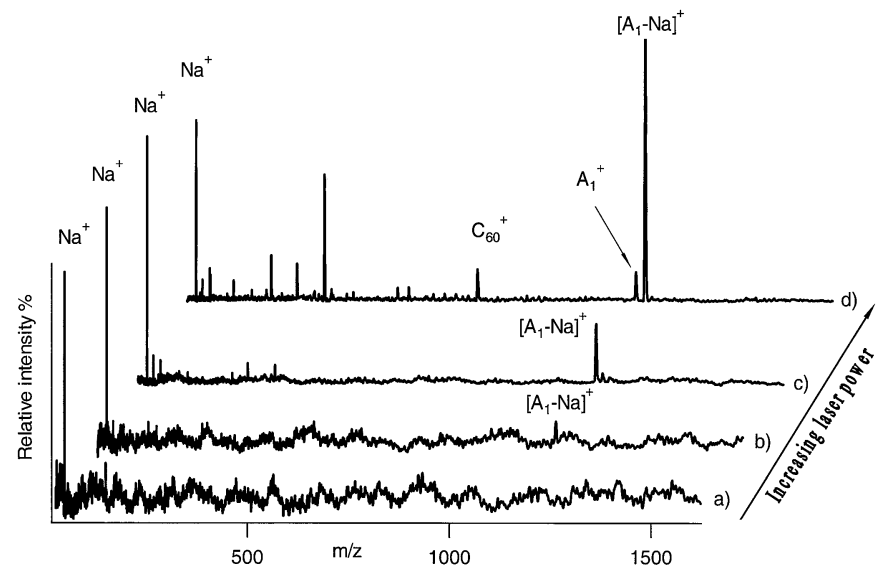

Figure 3. MALDI spectra recorded at increasing laser fluence (a-d). Analyte 1: DCTB (1:1000) layered on top of $\mathrm{NaBr}$. All spectra have been normalized to the respective base peak.

fluence applied to the activation of the target, in order to obtain insight into the mechanistic aspects governing ion formation [31]. A representative set of data is given in Figure 3 for the analysis of Analyte 1 using a $\mathrm{NaBr}$ salt layer.

The first ion observed upon increasing the laser fluence is the metal cation $\mathrm{Na}^{+}(m / z 23)$. Neither cation attachment at $\mathrm{m} / \mathrm{z} 1137$ nor the molecular cation radical $(\mathrm{m} / \mathrm{z} 1114)$ could be detected at low fluences (Figure 3a). To ensure that this finding was not caused by incomplete coverage of the target holder, the same sample area was activated at a higher laser fluence, whereby all the expected ions could be observed. At slightly higher fluences the signal corresponding to the attachment appears at $\mathrm{m} / \mathrm{z} 1137$ (Figure $3 \mathrm{~b}$ ) and becomes more abundant as the laser fluence is further increased (Figure 3c). This succession of events is probably caused by the minuscule amounts of analyte on the target. Only after sufficient amounts of the analyte were desorbed into the gas phase could attachment efficiently occur. Eventually, at even higher fluences, the molecular cation radical of the analyte is formed (Figure 3d). Experiments with other salt layers gave similar results. Detailed MALDI investigations into the cationization of biological samples [32] and polymers [33] have provided ample evidence for metal complexation to occur predominantly in the gas phase, rather than reflecting the desorption of preformed ions from the solid. In recent MALDI experiments it has been possible to distinguish gas phase complexation and ion preformation for cationized crown ethers [34]. Although the preformation of ions cannot be ruled out entirely in the present case, the method of sample preparation would not necessarily promote the preformation of the metal adduct ions, as the analyte/matrix layer was applied on top of the dried salt layer by using a solvent (toluene) in which the alkali salts have only low solubility. The finding that the molecular analyte ion is only observed at relatively high laser fluences indicates that metal attachment, as achieved in the present experiments, is in fact the "softer" approach towards ion formation.
Furthermore, none of the spectra contained metaladduct fragments composed of the metal ion and the fullerene ligand or moieties thereof. This can be taken as a further indication of a rather "soft" formation of the metal ion/analyte complex. If fragmentation of the complex occurred, the bare metal ion signal would represent the only discernible product of this decomposition. The most likely route of the molecular ion formation is by electron transfer from the ionized matrix and/or possible fragments thereof [35-37]. Matrix derived ions were always observed in spectra showing the molecular analyte ion. Although the ionization energy of DCTB is not known, comparison with structurally related molecules suggests that charge transfer from the ionized matrix should be energetically feasible.

\section{Metal Ion Attachment Competition}

To investigate the competition between the alkali metal ions for the attachment to a given analyte, all metals have been provided both in excess and as equimolar quantities of all individual salts in the bottom layer.

Along with the metal ion attachment, the intensities of the "naked" cations were also recorded in these spectra. In all cases the relative abundances of the bare metal cations followed the same trend, namely: $[\mathrm{Cs}]^{+}>$ $[\mathrm{Li}]^{+}>[\mathrm{Rb}]^{+}>[\mathrm{K}]^{+}>[\mathrm{Na}]^{+}$. Except for $\mathrm{Li}^{+}$, this sequence of bare metal ion intensities would be expected considering the lattice energies of the crystals [30]. The larger the cation, the smaller the lattice energy and hence the easier is the release of the cation from the crystal. The "abnormal" behavior of $\mathrm{Li}^{+}$cation is probably related to its very high hygroscopicity. During the sample preparation using Li-salts, it was occasionally observed that the salt would not simply dry when applied to the heated sample holder, but would smear out when brought to room temperature, probably caused by the incorporation of atmospheric water. The high hydration energies [30] of the small $\mathrm{Li}^{+}$cations could result in a much more pronounced hydration 

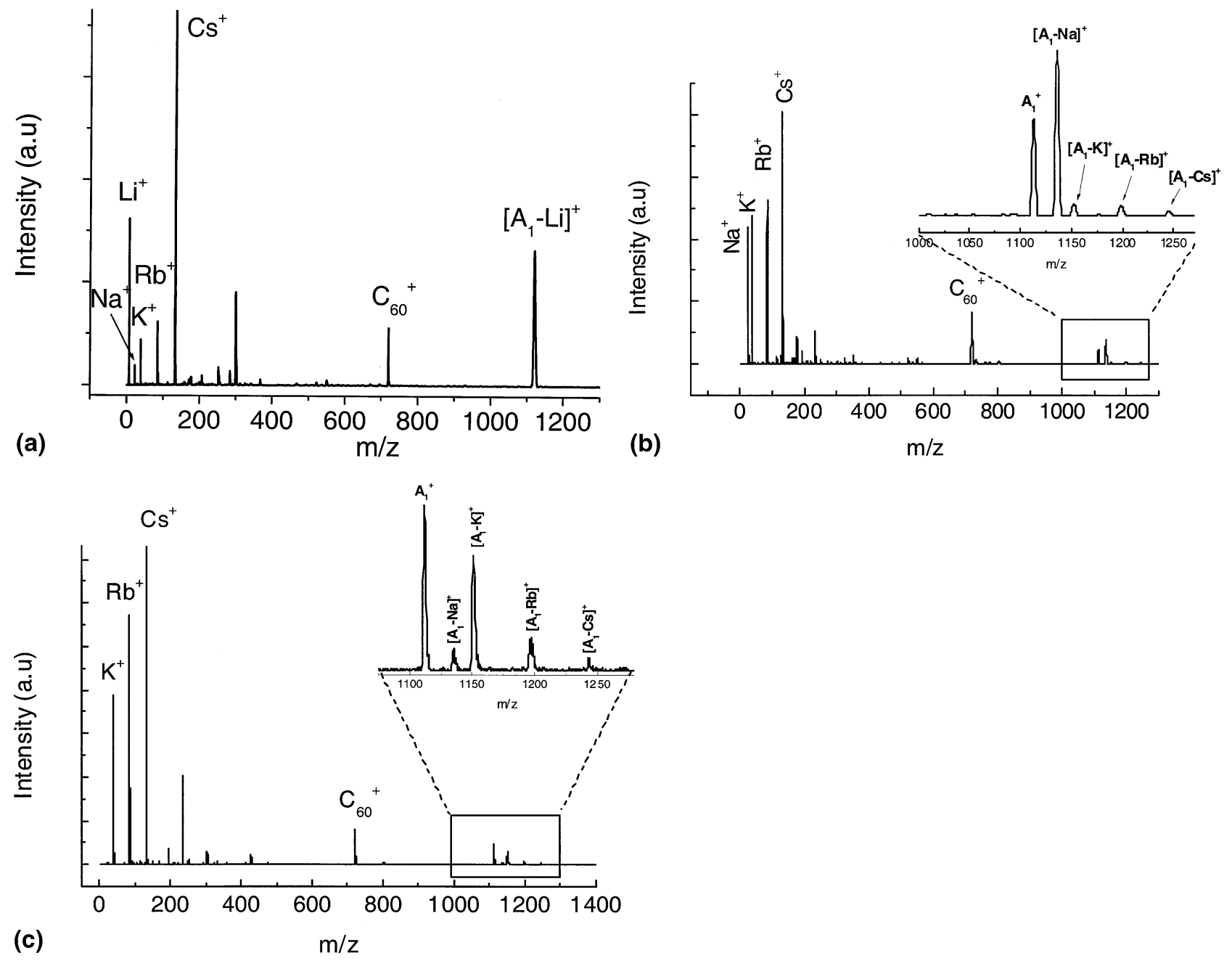

Figure 4. MALDI spectra of Analyte 1 with equimolar alkali chloride mixtures. Metal ion component in the salt layer: (a) All alkali cations $\mathrm{Li}^{+}$to $\mathrm{Cs}^{+}$, (b) $\mathrm{Na}^{+}$to $\mathrm{Cs}^{+}\left(\mathrm{Li}^{+}\right.$missing), (c) $\mathrm{K}^{+}$to $\mathrm{Cs}^{+}\left(\mathrm{Li}^{+}\right.$and $\mathrm{Na}^{+}$missing).

than for the other metal ions. Arguably, the actual extraction energy from the hydrated crystal layer should be much less than the energy necessary to extract a $\mathrm{Li}^{+}$cation from an ideal crystal.

Initially a salt layer was used that contained all alkali metal chlorides (Figure 4a). The attachment of $\mathrm{Li}^{+}$was that most abundantly observed, with only minor attachment of the other metals. To determine the attachment behavior of the other cations, the experiment was conducted without the $\mathrm{LiCl}$ component (Figure $4 \mathrm{~b}$ ). The most intense attachment was now with $\mathrm{Na}^{+}$followed by $\mathrm{K}^{+}, \mathrm{Rb}^{+}$, and $\mathrm{Cs}^{+}$. Finally, the use of a salt layer composed of only $\mathrm{K}, \mathrm{Rb}$, and $\mathrm{Cs}$ chlorides in equimolar amounts (Figure 4c) confirmed the $[\mathrm{A}-\mathrm{Li}]^{+}>[\mathrm{A}-\mathrm{Na}]^{+}$ $>[\mathrm{A}-\mathrm{K}]^{+}>[\mathrm{A}-\mathrm{Rb}]^{+}>[\mathrm{A}-\mathrm{Cs}]^{+}$order. The same sequence was also obtained with Analyte 2 .

The abundances for the bare metal ions $\mathrm{Na}^{+}, \mathrm{K}^{+}$, $\mathrm{Rb}^{+}$, and $\mathrm{Cs}^{+}$is thus exactly the reverse of the abundances of the respective analyte/metal ion complexes. These data clearly indicate that the efficiency of the cation attachment is not primarily linked to the gas phase concentration of the metal ion. This observation is also true for $\mathrm{Li}^{+}$. In experiments with $\mathrm{Li}^{+}$as a minor component in the salt layer, leading to a less abundant $\mathrm{Li}^{+}$signal compared with other bare alkali metal ions, analyte complexation with $\mathrm{Li}^{+}$was still observed as the most prominent attachment. Similar observations have been made during the MALDI analysis of dendrimers, where certain minute metal ion impurities led to abundant complexation [38]. These findings clearly underline that the most important factor determining the attachment ion intensity relates to the intrinsic metal ion affinity of the analyte, rather than to the concentration of the cation in the MALDI plume.

Indeed, the attachment order follows the charge density of the metal ion and thus its polarization power, as well as its electron affinity. These quantities strongly influence the cation affinity to the derivatized fullerenes. $\mathrm{Li}^{+}$is the smallest ion with the highest charge density and electron affinity. It thus possesses the highest polarization power considering purely electrostatic interactions. It is therefore reasonable to assume 
that $\mathrm{Li}^{+}$will have the highest affinity towards the fullerene derivatives. In fact, the observed intensity order corresponds to the intrinsic affinity of the bare alkali metal ions that was, for instance, observed for the complexation with crown ethers in the gas phase, as opposed to complexation in solution which follows a different sequence caused by solvation of the ions [34, 39].

The experiments just discussed offer the relevant metal ions simultaneously through the salt layer and therefore provide direct insight into the different attachment efficiencies. However, the same metal ion sequence has been corroborated by a careful comparison of individual experiments using only single salt layers (Figure 2). Caused by inherent reproducibility problems, the absolute intensities of ions obtained in MALDI can differ quite substantially from experiment to experiment. To allow direct comparison of individual ion abundances in different experiments, the intensity of the metal ion attachment signal was normalized to all the major analyte-derived signals. Bearing in mind that the two major routes of the analyte molecule include formation of the adduct ion, ionization through electron transfer and possible subsequent fragmentation into $\mathrm{C}_{60}^{+}$, the attachment fraction $\mathbf{F}$ is established as follows:

$$
\mathrm{F}=\frac{[\mathrm{AY}]^{+}}{[\mathrm{AY}]^{+}+[\mathrm{A}]^{+}+\left[\mathrm{C}_{60}\right]^{+}}
$$

where $[\mathrm{A}]^{+}$represents the intensity of the molecular ion of the analyte and $[\mathrm{AY}]^{+}$the intensity of the attached species. $\left[\mathrm{C}_{60}\right]^{+}$compensates for the loss of initially formed molecular ions $\mathrm{A}^{+}$by fragmentation. The attachment fraction $\mathbf{F}$ represents a normalization which links all the major ions derived from the analyte and produced by both ion formation routes. It thus allows the comparison of different experiments independent of the respective absolute ion intensities. The attachment fraction $\mathbf{F}$ showed only slight deviations over a relatively large range of laser fluences, while the intensity of the $\mathrm{C}_{60}^{+}$fragment increases as a function of the laser fluence (due to fragmentation). This behavior indicates that the $\mathrm{C}_{60}^{+}$fragment arises in fact from dissociation of the molecular analyte ion rather than from decompositions of the metal ion/analyte complex.

Figure 5 displays the attachment fraction $\mathbf{F}$ obtained for the individual alkali metals as derived from analysis of the single salt layer experiments. Over a wide range of activating laser fluences, the same metal ion sequence is obtained as $\mathbf{F}[\mathrm{A}-\mathrm{Li}]^{+}>\mathbf{F}[\mathrm{A}-\mathrm{Na}]^{+}>\mathbf{F}[\mathrm{A}-\mathrm{K}]^{+}$. The attachment fractions obtained for $\mathrm{Rb}^{+}$and $\mathrm{Cs}^{+}$also follow the same sequence established above, but were too low to allow a meaningful display in Figure 5.

\section{Analyte Competition for Metal Ion Attachment}

In order to reveal the competition between two analytes for the attachment of a particular metal ion, the two analytes were studied simultaneously. To maintain a

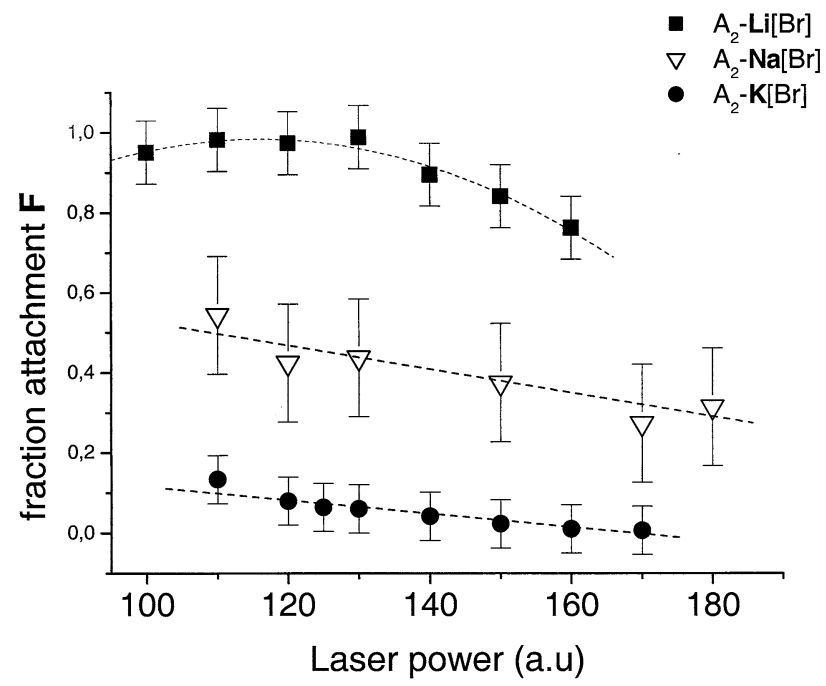

Figure 5. Attachment fraction, $\mathbf{F}$ (as defined in the text), versus laser fluence as obtained from the analysis of single salt layer MALDI experiments with Analyte 2 using as bottom layer: Filled square - $\mathrm{LiBr}$, open inverted triangle - $\mathrm{NaBr}$, filled circle - $\mathrm{KBr}$. The error bars give an estimated standard deviation (see Experimental section).

1000:1 ratio of matrix to analyte, the individual analyte solutions were prepared to a 500:1 ratio and then mixed together giving an overall matrix to analyte ratio of 1000:1.

First, the two analytes were investigated as equimolar mixture without use of a salt layer in order to elucidate possible differences regarding their behavior in the desorption/ionization process. Both molecular cation radicals were observed in almost the same abundances over a wide laser fluence range, which established that the overall efficiency for desorption and electron transfer ionization is for both analytes roughly the same. Therefore, when considering the metal attachment behavior, discrimination against one of the analytes during desorption can be discounted. Lithium and sodium were chosen as metals for this part of the investigation, representing the two metals that display the highest efficiency for the attachment while being considerably different in their behavior. For each metal, the chloride, bromide and iodide were studied, respectively.

Analyte competition for sodium ion attachment. A typical spectrum with a NaBr layer is shown in Figure 6a. The molecular cation radicals of both analytes have been observed, as well as the $\mathrm{Na}^{+}$adduct ions. The important observation is that at all laser fluences the $\mathrm{Na}^{+}$adduct intensities follow $\left[\mathrm{A}_{1}-\mathrm{Na}\right]^{+}>\left[\mathrm{A}_{2}-\mathrm{Na}\right]^{+}$, while the molecular ion abundances are reversed, $\left[\mathrm{A}_{1}\right]^{+}<\left[\mathrm{A}_{2}\right]^{+}$. Very similar results are also obtained with $\mathrm{NaCl}$ used as the salt layer. The intensity ratio of the metal ion attachment to Analyte 1 and Analyte $2,\left[\mathrm{~A}_{1}-\mathrm{Y}\right]^{+} /\left[\mathrm{A}_{2}-\mathrm{Y}\right]^{+}$, is given in Figure 7 as a function of the laser fluence.

The efficiency of the sodium attachment is distinctly different for both analytes; an observation which is 

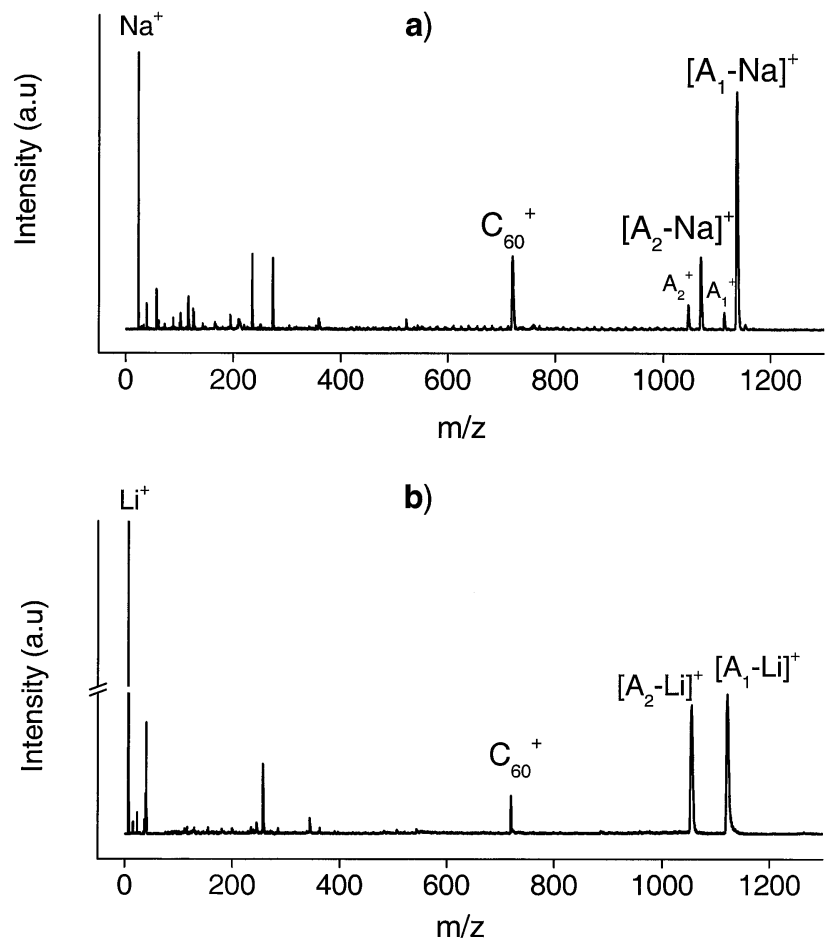

Figure 6. MALDI spectra of the equimolar mixture of both analytes $\left(\mathrm{A}_{1}: \mathrm{A}_{2}: \mathrm{DCTB}=1: 1: 1000\right)$ on top of $(\mathbf{a}) \mathrm{NaBr}$ and $(\mathbf{b}) \mathrm{LiBr}$ salt layer.

most likely caused by the structural difference of the two ligands (Figure 1a and b). Analyte 1 is obviously much more attractive for sodium ion complexation than Analyte 2. The ligand of Analyte 1 can be regarded as ring-opened crown ether, providing many potential sites for interaction with the metal ion. The heteroatom arrangement of the ligand in Analyte 2 is clearly less favored in this competition. The sodium ion attachment thus shows selectivity in favor of the crown ether-like ligand.

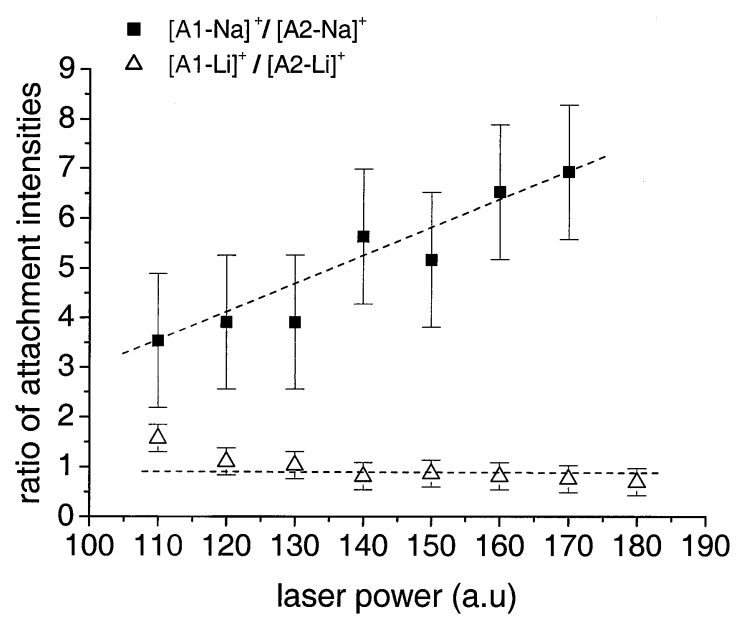

Figure 7. The filled square $-\left[\mathrm{A}_{1}-\mathrm{Na}^{+}\right] /\left[\mathrm{A}_{2}-\mathrm{Na}^{+}\right]$and the open triangle $-\left[\mathrm{A}_{1}-\mathrm{Li}^{+}\right] /\left[\mathrm{A}_{2}-\mathrm{Li}^{+}\right]$ratios by MALDI of the equimolar mixture on the corresponding single salt layers. (The dashed lines are meant to guide the eye and have no physical meaning).
The other intriguing finding derived from this experiment is the inverse intensity order in which the molecular cation radicals are observed. The more efficient, and therefore enhanced, the metal ion attachment process, the lower is the intensity of the molecular ion, thus clearly reflecting the competition in the formation of these ions. A similar trend is evident in the mass spectra obtained by using one analyte and all metal ions simultaneously (Figure 4). Excluding lithium (Figure $4 \mathrm{~b})$ and then sodium (Figure 4c) from the salt layer gradually increases the formation of the molecular cation radical, as those metals remaining on the target become less efficient in the attachment reaction. The rationalization of these findings is provided within the framework of the ion formation mechanisms in MALDI [31]. The neutral analyte molecule, represents the least abundant component that is transferred into the gas phase upon laser activation. Analyte ions may be formed predominantly via two competing reactions occurring in the fast expanding material plume. First, molecular cation radicals may result from charge exchange between the analyte and the ionized DCTB matrix and/or ionic fragments thereof $[15,16]$. Second, cationization may occur through the interaction of bare metal ions with the analyte. The cross sections of each of these competing reactions in a given situation will determine which ions are eventually observed. If metal attachment is less efficient, more analyte molecules may ionize via the electron transfer route. These considerations are in line with the present observation that a less efficient metal ion attachment, such as the sodiation of Analyte 2, is accompanied by a comparatively more pronounced formation of the molecular ion, which is clearly formed more abundantly for Analyte 2 than for Analyte 1. A particularly intriguing example of how alkali metal coordination dictates the reactivity towards fragmentation can be found in reference [40].

Analyte competition for lithium ion attachment. $\mathrm{Li}^{+}$attachment is observed very abundantly for both analytes using $\mathrm{LiCl}, \mathrm{LiBr}$, and $\mathrm{LiI}$ as the salt layer, respectively (Figure 6b). The molecular cation radicals of Analyte 1 $(\mathrm{m} / \mathrm{z}$ 1114) and Analyte $2(\mathrm{~m} / \mathrm{z}$ 1047) were only observed with the $\mathrm{LiCl}$ salt layer, but not in the case of $\mathrm{LiBr}$ and LiI. In line with the preceding discussion, this observation seems to indicate that $\mathrm{Li}^{+}$attachment applying $\mathrm{LiBr}$ and $\mathrm{LiI}$ is so efficient that the molecular ion formation is practically suppressed. Furthermore, a counter ion effect seems to operate, as the use of the chloride reduces the metal attachment and allows the electron transfer process to compete. The effects caused by the counter ions will be discussed in more detail below.

When comparing the $\mathrm{Li}^{+}$attachment to both analytes it is striking to note that the signal for $\left[\mathrm{A}_{1}-\mathrm{Li}\right]^{+}$is nearly as intense as the $\left[\mathrm{A}_{2}-\mathrm{Li}\right]^{+}$peak, leading to a $\left[\mathrm{A}_{1}-\mathrm{Li}\right]^{+} /$ $\left[\mathrm{A}_{2}-\mathrm{Li}\right]^{+}$ratio close to one (Figure 7 ). The reactivity pattern for $\mathrm{Li}^{+}$is thus distinctly different from the one observed for $\mathrm{Na}^{+}$. The structural differences of both 

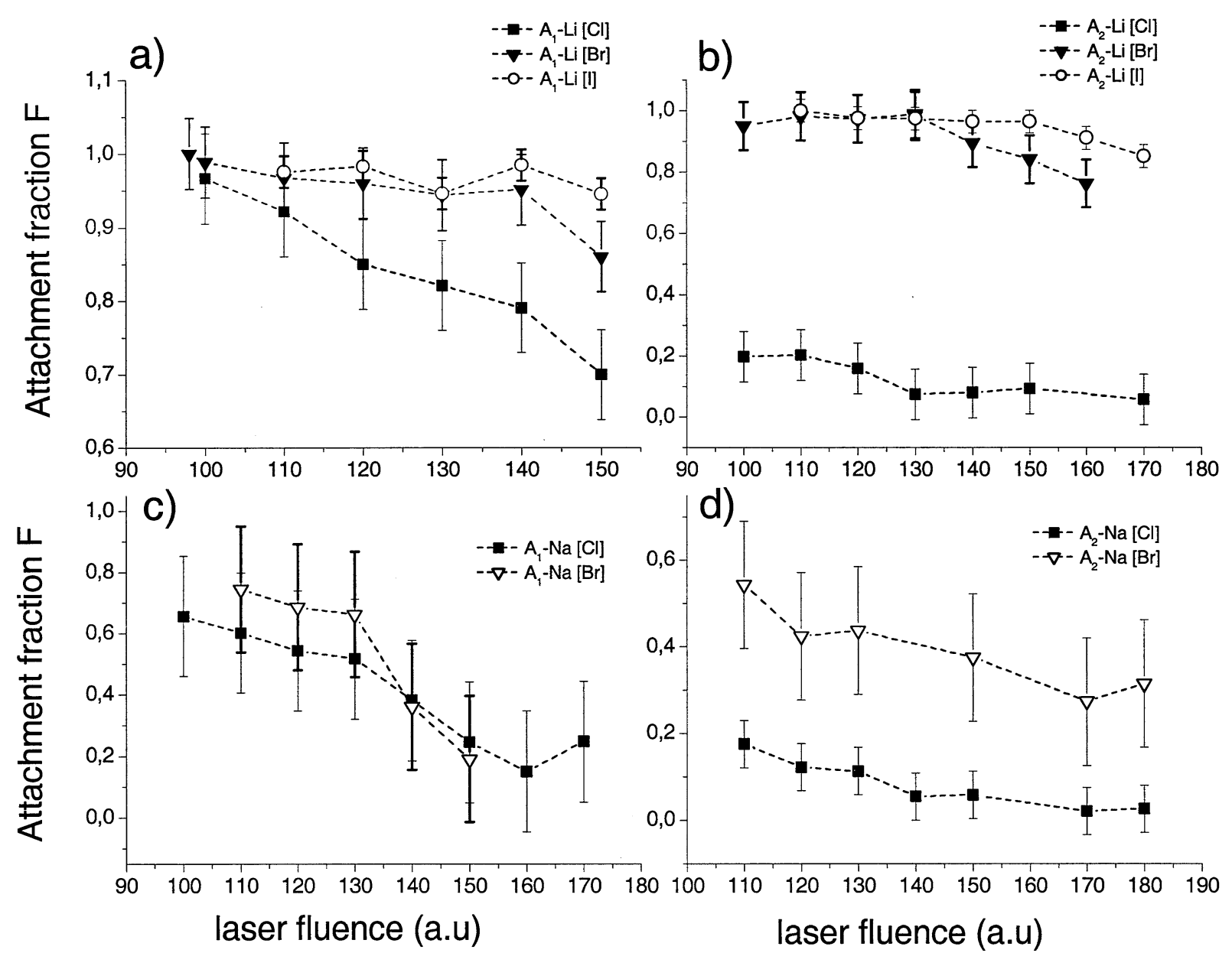

\begin{abstract}
Figure 8. Counter-ion effect on the attachment fraction, F, for the complexation of the Analyte 1 or Analyte 2 with $\mathrm{Li}^{+}$or $\mathrm{Na}^{+}$, as a function of the laser fluence. (The dashed lines are meant to guide the eye and have no physical meaning). Attachment fraction of $\left[\mathrm{A}_{1}-\mathrm{Li}\right]^{+}$(a) derived from MALDI of Analyte 1 using filled square - $\mathrm{LiCl}$, filled inverted triangle - $\mathrm{LiBr}$, and open circle - LiI, respectively. Attachment fraction of $\left[\mathrm{A}_{2}-\mathrm{Li}\right]^{+}(\mathbf{b})$ derived from MALDI of Analyte 2 using filled square - $\mathrm{LiCl}$, filled inverted triangle - $\mathrm{LiBr}$, and open circle - LiI, respectively. Attachment fraction of $\left[\mathrm{A}_{1}-\mathrm{Na}\right]^{+}$(c) derived from MALDI of Analyte 1 using filled square - $\mathrm{NaCl}$ and open triangle - $\mathrm{NaBr}$, respectively. Attachment fraction of $\left[\mathrm{A}_{2}-\mathrm{Na}\right]^{+}(\mathbf{d})$ derived from MALDI of Analyte 2 using filled square $-\mathrm{NaCl}$ and open triangle - $\mathrm{NaBr}$.
\end{abstract}

analytes, which caused a clear differentiation for the attachment with $\mathrm{Na}^{+}$, are insufficient to achieve selectivity in the attachment with $\mathrm{Li}^{+}$. The $\mathrm{Li}^{+}$cation with its high charge density is obviously more reactive and attaches to both analytes with the same high efficiency. In fact, the observed nearly one-to-one ratio of lithiated Analyte 1 and Analyte 2 (Figure 6) is simply a reflection of the abundances of both neutral analytes in the plume, as desorbed from the initial equimolar mixture on the target.

In turn, $\mathrm{Na}^{+}$displays a lower affinity and thus reduced reactivity with both analytes and therefore reacts more selectively. Such an inverse relationship between affinity/reactivity versus selectivity is a common phenomenon in chemistry.

\section{Counter Ion Effects}

This section investigates possible effects caused by the counter ions of the alkali metals on the extent to which metal attachment has been observed. The data have been derived from the MALDI analysis of the individual analyte/matrix layer on a single salt layer. The attachment of $\mathrm{Li}^{+}$and $\mathrm{Na}^{+}$to the individual analytes has been investigated using $\mathrm{Cl}^{-}, \mathrm{Br}^{-}$, and $\mathrm{I}^{-}$as the counter ions. Varying the incoming laser fluence, the metal ion attachment is studied as a function of the counter ion by making use of the attachment fraction, $\mathbf{F}$, as introduced earlier.

For different counter ions, Figure 8a and b display the $\mathrm{Li}^{+}$attachment fractions in reactions with Analytes 1 and 2, respectively. The higher the value of the fraction, the more important is the attachment of the cation compared to competitive processes. The attachment of $\mathrm{Li}^{+}$to Analyte 1 is practically unaffected when changing the LiI salt layer to $\mathrm{LiBr}$ (Figure 8a). As discussed earlier, the attachment of $\mathrm{Li}^{+}$is most efficient, so that the attachment fraction is close to one. However, the use of $\mathrm{LiCl}$ clearly leads to slightly lower attachment 
fractions. This effect becomes even more pronounced for the $\mathrm{Li}^{+}$attachment to Analyte 2 (Figure 8b). Note the different scales for the fraction, $\mathbf{F}$, in each of the figures. Again, there is little difference for the application of LiI and $\mathrm{LiBr}$, however, the $\mathrm{LiCl}$ salt layer leads to a dramatic reduction of the $\mathrm{Li}^{+}$attachment to Analyte 2 .

The attachment of $\mathrm{Na}^{+}$to Analytes 1 and 2 is shown in Figure $8 \mathrm{c}$ and $\mathrm{d}$, respectively. In line with earlier findings, the lower affinity to both analytes is clearly reflected by the lower fractions obtained compared to the experiments with $\mathrm{Li}^{+}$(Figure 8a and b). The most pronounced counter ion effect is again evident for the attachment with Analyte 2 (Figure 8d), where the use of $\mathrm{NaCl}$ clearly reduces the attachment of $\mathrm{Na}^{+}$. As a general trend, the use of metal chlorides, as opposed to the application of bromides and iodides, results in lower cation attachment fractions.

The extent to which the metal ion attachment occurs is thus affected by the counter anion. Reducing the size of the anionic salt component reduces in the present case the cation attachment. Similar findings have been noted for the cationization of polymers [41]. This counter ion effect correlates roughly with the lattice energies of the salt crystals [30]. The lattice energy decreases for larger counter ions. This makes the break-up of the crystal energetically more feasible and increases the concentration of cations in the gas phase. Hence, for larger counter ions, the metal attachment is enhanced. These findings seem to indicate that the availability of the metal ion in the gas phase is of influence on the extent to which a particular metal ion attaches to the analyte. A reduction of the metal ion availability could also come through recombination with the counter ion. If anionic counter ions are sputtered from the target, recombination with the metal ions could take place prior to the recapture of the anion by the positively charged target holder. Since the metal ion is more strongly bound to smaller counter ions, the recombination becomes energetically more attractive. The resulting reduced availability of the metal ion for attachment could therefore also contribute to the observed counter ion effect.

\section{Conclusions}

Alkali metal ion attachment to derivatized, amphiphilic fullerenes has been investigated using MALDI mass spectrometry. Circumstantial evidence is presented that complexation occurs through interaction of the metal ion with the ligand rather than with the fullerene core. The metal ion complexation competes efficiently with the formation of the molecular analyte ion by electron transfer ionization. In fact, it is found that ion formation by metal ion attachment is energetically less demanding in terms of target activation. Metal ion complexed analyte ions have been observed more abundantly, displaying less fragmentation and thus implying an overall gentler route to desorption and ionization of the sample.
The reactivity order of the metal ions and the analytes has been established. The extent by which alkali ion attachment has been observed increases with decreasing size of the metal ion. This feature is rationalized in terms of the variation of the charge density of the metal ion and, connected with this, the ability of the metal ion to polarize and interact electrostatically with the heteroatoms of the fullerene linked ligand. The structure of the fullerene ligand is also of profound influence. More efficient complexation has been observed with a crown ether-like ligand as opposed to a ligand which featured one amide and two ester groups. The halide counter ion is a further influential factor determining the metal ion complexation in that less cation attachment resulted with smaller counter ions. Mechanisms by which the counter ion may affect the availability of the metal ion for complexation have been discussed.

\section{Acknowledgments}

The authors are grateful to Dr. A. Hoxha for helpful advice and thank Professor T. J. Kemp for the critical reading of the manuscript. BL thanks the Fonds National de la Recherche Scientifique (Belgium) for a research associate position. DF and BL acknowledge financial support by the Direction de la Recherche Scientifique de la Communauté Française de Belgique through an Action de Recherche Concertée (A.R.C.). The work at Warwick was made possible through funding by the Leverhulme Trust and the EPSRC.

\section{References}

1. Hirsch, A. The Chemistry of the Fullerenes. G. Thieme Verlag: Stuttgart and New York, 1994.

2. Taylor, R. Lecture Notes on Fullerene Chemistry: A Handbook for Chemists. Imperial College Press: London, 1998.

3. Kaddish, K. M.; Ruof, R. S., Eds. In Fullerenes: Chemistry, Physics, and Technology. John Wiley and Sons: New York, 2000.

4. Wilson, S. R. Biological Aspects of Fullerenes. In Fullerenes: Chemistry, Physics, and Technology. John Wiley and Sons: New York, 2000; Chap X p 437.

5. Wilson, L. J. The Electrochemical Society Interface; Winter 1999, p. 24

6. Guldi, D. M.; Tian, Y.; Fendler, J. H.; Hungerbühler, H.; Asmus, K.-D. Stable Monolayers and Langmuir-Blodgett Films of Functionalized Fullerenes. J. Phys. Chem. 1995, 99, 17673-17676.

7. Cardullo, F.; Diederich, F.; Echegoyen, L.; Habicher, T.; Fayaraman, J.; Leblanc, R. M.; Stoddart, J. F.; Wang, S. Stable Langmuir and Langmuir-Blodgett Films of Fullerene-Glycodendron Conjugates. Langmuir 1998, 14, 1955-1959.

8. Van Berkel, G. J. In Electrospray Ionization Mass Spectrometry: Fundamentals, Instrumentation, and Applications; Cole, R. B., Ed.; John Wiley, New York, 1997; Chap II p 65.

9. Dupont, A.; Gisselbreht, J.-P.; Leize, E.; Wagner, L.; Van Dorsselaer, A. Electrospray Mass-Spectrometry of Electrochemically Ionized Molecules-Application to the Study of Fullerenes. Tetrahedron Lett. 1994, 35, 6083-6086.

10. Liu, T.-Y.; Shiu, L.-L.; Luh, T.-Y.; Her, G.-R. Direct Analysis of $\mathrm{C}_{-60}$ and Related Compounds with Electrospray Mass-Spectrometry. Rapid Commun. Mass Spectrom. 1995, 9, 93-96.

11. Barrow, M. P.; Feng, X.; Wallace, J. I.; Boltalina, O. V.; Taylor, R.; Derrick, P. J.; Drewello, T. Characterization of Fullerenes 
and Fullerene Derivatives by Nanospray. Chem. Phys. Lett. 2000, 330, 267-274.

12. Osterodt, J.; Vögtle, F. $\mathrm{C}_{61} \mathrm{Br}_{2}$ : A New Synthesis of Dibromomethanofullerene and Mass Spectrometric Evidence of the Carbon Allotropes $C_{121}$ and $C_{122}$. Chem. Commun. 1996, 547548.

13. Brune, D. C. Elemental Sulfur as a Matrix for Mass Spectrometry of Photosynthetic Pigments and Fullerenes. Rapid Commun. Mass Spectrom. 1999, 13, 384-389.

14. Vasil'ev, Y.; Wallis, D.; Nüchter, M.; Ondruchka, B.; Lobach, A.; Drewello, T. From Major to Minor and Back-A Decisive Assessment of $\mathrm{C}_{60} \mathrm{H}_{36}$ with Respect to the Birch Reduction of $\mathrm{C}_{60}$. Chem. Commun. 2000, 1233-1234.

15. Ulmer, L.; Mattay, J.; Torres-Garcia, H. G.; Luftmann, H. Letter: The Use of 2-[(2E)-3-(4-tert-butylphenyl)-2-methylprop-2-enylidene]malononitrile as a Matrix for Matrix-Assisted Laser Desorption/Ionization Mass Spectrometry. Eur. J. Mass Spectrom. 2000, 6, 49-52.

16. Brown, T.; Clipston, N. L.; Simjee, N.; Luftmann, H.; Hungerbühler, H.; Drewello, T. Matrix-Assisted Laser Desorption/ Ionization of Amphiphilic Fullerene Derivatives. Int. J. Mass. Spectrom. 2001, 210/211, 249-263.

17. Nielen, M. W. F. Maldi Time-of-Flight Mass Spectrometry of Synthetic Polymers. Mass Spectrom. Rev. 1999, 18, 309-344.

18. Barrow, M. P.; Drewello, T. Significant Interferences in the Post Source Decay Spectra of Ion-Gated Fullerene and Coalesced Carbon Cluster Ions. Int. J. Mass Spectrom. 2000, 203, 111-125.

19. Clipston, N. L.; Brown, T.; Vasil'ev, Y. V.; Barrow, M. P.; Herzschuh, R.; Reuther, U.; Hirsch, A. Laser-Induced Formation, Fragmentation, Coalescence, and Delayed Ionization of the $\mathrm{C}_{59} \mathrm{~N}$ Heterofullerene. J. Phys. Chem. A 2000, 104, 9171-9179.

20. Vasil'ev, Y. V.; Absalimov, R. R.; Nasibullaev, S. K.; Lobach, A. S.; Drewello, T. Formation and Characterization of LongLived Negative Molecular Ions of $\mathrm{C}_{60} \mathrm{H}_{18}$. J. Phys. Chem. A 2001, 105, 661-665.

21. Boorum, M. M.; Vasil'ev, Y. V.; Drewello, T.; Scott, L. T. Groundwork for a Rational Synthesis of $\mathrm{C}_{60}$ : Cyclodehydrogenation of a $\mathrm{C}_{60} \mathrm{H}_{30}$ Polyarene. Science 2001, 294, 828-831.

22. Whaley, T. P. Sodium, Potassium, Rubidium, Cesium, and Francium. In Comprehensive Inorganic Chemistry; Bailar, J. C.; Emeléus, H. J.; Sir Nyholm, R.; Trorman-Dickenson, A. F., Eds. Pergamon Press: Oxford, 1973, Vol. I p 406.

23. Axelsson, J.; Hoberg, A.-M.; Waterson, C.; Myatt, P.; Shield, G. L.; Varney, J.; Haddleton, D. M.; Derrick, P. J. Improved Reproducibility and Increased Signal Intensity in MatrixAssisted Laser Desorption/Ionization as a Result of Electrospray Sample Preparation. Rapid Commun. Mass Spectrom. 1997, 11, 209-213.

24. Hoberg, A.-M.; Haddelton, D. M.; Derrick, P. J. Evidence for Cationization of Polymers in the Gas Phase During MatrixAssisted Laser Desorption/Ionization. Eur. Mass Spectrom. 1997, 3, 471-473.

25. Wilson, S. R.; Wu, Y. J. Crown-Ether Fulleroids and Their Detection in Solution by Electrospray MS. Chem. Soc. Chem. Commun. 1993, 784-786.

26. Huang, Y.; Freiser, B. S. Synthesis of Bis(Buckminsterfullerene)Nickel Cation, $\mathrm{Ni}\left(\mathrm{C}_{60}\right)_{2}^{+}$, in the gas phase. J. Am. Chem. Soc. 1991, 113, 8186-8187.
27. Huang, Y.; Freiser, B. S. Externally Bound Metal-Ion Complexes of Buckminsterfullerene, $\mathrm{MC}_{60}^{+}$, in the Gas Phase. J. Am. Chem. Soc. 1991, 113, 9418-9419.

28. Buchanan, J. W.; Grieves, G. A.; Reddic, J. E.; Duncan, M. A Novel Mixed Ligand Sandwich Complexes: Competitive Binding of Iron with Benzene, Coronene, and $\mathrm{C}_{60}$. Int. J. Mass Spectrom. 1999, 182/183, 323-333.

29. de Vries, J.; Steger, H.; Kamke, B.; Menzel, C.; Weisser, B.; Kamke, W.; Hertel, I. V. Single-Photon Ionization of $C_{60}$ Fullerene and $C_{70}$ Fullerene with Synchroton Radiation-Determination of the Ionization-Potential of $\mathrm{C}_{60}$. Chem. Phys. Lett. 1992, 188, 159-162.

30. Hart, A. W.; Beumel, O. F. Lithium and its Compounds. In Comprehensive Inorganic Chemistry; Bailar, J. C.; Emeléus, H. J.; Sir Nyholm, R.; Trorman-Dickenson, A. F., Eds. Pergamon Press: Oxford, 1973, Vol. I, pp 340-341.

31. Zenobi, R.; Knochenmuss, R. Ion Formation in MALDI Mass Spectrometry. Mass Spectrom. Rev. 1998, 17, 337-366.

32. Belov, M. E.; Myatt, C. P.; Derrick, P. J. Chemical Ionization of Neutral Peptides Produced by Matrix-Assisted Laser Desorption. Chem. Phys. Lett. 1998, 284, 412-418.

33. Mowat, I. A.; Donovan, R. J.; Maier, R. R. J. Enhanced Cationization of Polymers Using Delayed Ion Extraction with Matrix-Assisted Laser Desorption/Ionization. Rapid Commun. Mass Spectrom. 1997, 11, 89-98.

34. Calba, P.-J.; Muller, J.-F.; Schmitt-Dubessy, M.-F.; Joly, J.-P.; Chapleur, Y. Scope and Limitation of Matrix-Assisted Laser Desorption/Ionization Fourier Transform Mass Spectrometry for Testing the Complexing Behavior of Two Crown Ethers Derived from D-Hexopyranosides. Eur. J. Mass Spectrom. 2002, 8, 41-51.

35. Juhasz, P.; Costello, C. E. Generation of Large Radical Ions from Oligometallocenes by Matrix-Assisted Laser Desorption Ionization. Rapid Commun. Mass Spectrom. 1993, 7, 343-351.

36. McCarley, T. D.; McCarley, R. L.; Limbach, P. A. Electron Transfer Ionization in Matrix Assisted Laser Desorption/Ionization Mass Spectrometry. Anal. Chem. 1998, 70, 4376-4379.

37. Macha, S. M.; McCarley, T. D.; Limbach, P. A. Influence of Ionization Energy on Charge-Transfer Ionization in MatrixAssisted Laser Desorption/Ionization Mass Spectrometry. Anal. Chim. Acta 1999, 397, 235-245.

38. Mowat, I. A.; Donovan, R. J.; Bruce, M.; Feast, W. J.; Stainton, N. M. Matrix Assisted Laser Desorption/Ionization Mass Spectrometry of Aryl Ester Dendrimers. Eur. Mass Spectrom. $1998,4,451-458$.

39. Lee, S.; Wittenbach, T.; von Helden, G.; Bowers, M. T. Gas Phase Conformations of $\mathrm{Li}^{+}, \mathrm{Na}^{+}, \mathrm{K}^{+}$, and $\mathrm{Cs}^{+}$complexed with 18-Crown-6. J. Am. Chem. Soc. 1995, 117, 10159-10160.

40. Cancilla, M. T.; Penn, S. G.; Caroll, J. A.; Lebrilla, C. B. Coordination of Alkali Metals to Oligosaccharides Dictates Fragmentation Behavior in Matrix Assisted Laser Desorption Ionization Fourier Transform Mass Spectrometry. J. Am. Chem. Soc. 1996, 118, 6736-6745.

41. Hoberg, A.-M.; Haddleton, D. M.; Derrick, P. J.; Jackson, A. T.; Scrivens, J. H. The Effect of Counter Ions in Matrix-Assisted Laser Desorption/Ionization of Poly(methyl methacrylate). Eur. Mass Spectrom. 1998, 4, 435-440. 